Article

\title{
Analysis of Driven Piles Using Local and Non-Local Models
}

\author{
Nicolas Sau ${ }^{1, *}$, José Medina-Mendoza ${ }^{1}$ and Antonia López-Higuera ${ }^{1}$ \\ 1 University of Sonora, Civil Engineering and Mines Department, Boulevard Encinas and Rosales, \\ Hermosillo, Mexico, 83000; nicolas.sau@unison.mx, jose.medina@unison.mx, antonia.lopez@unison.mx. \\ * Correspondence: nicolas.sau@unison.mx; Tel.: +52 6622592183, +52 6622794123.
}

\begin{abstract}
There exists a great potential for application in soil mechanics with the use of mechanistic models such as the finite element model, and other non-local models, such as the peridynamic model, since the soil mass can be modelled as a group of particles that interact with each other. In this work, we determine the bearing capacity of deep foundations where stresses are transmitted to deep deposits, which generally present better characteristics in terms of compressibility and shear strength. One of the main elements are driven piles, which must be previously designed, made and tested before their final use. In this work, a comparison with different methods is presented. The finite element method and the peridynamic model are used. Because the effect of consolidation was not taken into account, in these examples, the assumption is made that the structures are cemented in inert soil. Likewise, it is assumed that the water table has no influence on the simulations. Failure envelopes were observed where bonds between particles present high gradients of deformation and fracture. Bearing capacities were estimated and compared with those obtained from the Terzaghi and Meyerhof methods.
\end{abstract}

Keywords: foundations; soils; finite element method; peridynamics

\section{Introduction}

Soil is a complex material which, in order to be modeled, requires important hypotheses similar to those made in other branches of mechanics, such as continuity, homogeneity and isotropy. Due to these hypotheses, there are significant differences between theoretical models and reality. Therefore, the good judgment of the engineer and his experience play a key role. For the calculation of foundations there is a variety of solutions for different given problems, such as footings and piles. In the analysis and design of foundations it is necessary to choose the type of suitable model that represents the situation faced by the project engineer.

There are two major classes of foundations, surface and deep foundations. Piles are a type of a deep foundation, which are classified as friction piles and tip piles. These types of elements must be analyzed and tested prior to placement. For the calculation of superficial and deep foundations there are different theories developed mainly by Terzaghi, Meyerhof and Prandtl [1]. These types of models are the most accepted today by the engineer. However, given the rise of computers in recent years, there has been a tendency to use other methods of analysis such as the finite element.

Current models have limited applications to quasi-fragile structures such as rocks and soil. Existing models for quasi-fragile materials can be classified as continuous and discrete. The formulation of the finite element method is a remarkable advance in the modeling of civil engineering structures. However, problems appear when damage and discontinuities develop in these types of structures [2].

In an effort to correct the deficiencies of continuous models, the "peridynamic" model was proposed in 1998 by Silling. Stewart Silling proposed in 1998 the peridynamic term of the Greek word "peri" which means close. The equations in this type of formulation do not assume spatial differentiation of the field of displacements and allow discontinuities to arise as part of the solution 
[3]. This approach is also interpreted as a continuous adaptation of molecular dynamics models. Peridynamic theory is not local, where locality recovers as a special case. In addition, the peridynamic formulation can be considered as a generalization of the classical theory of elasticity.

Obviously, it is not correct to model soils as continuous materials using smeared cracks as is done in many material models such as in concrete and rock models, however, soil is far from being a continuous material, where the mechanisms of general failure can be compared with the propagation of cracks in quasi-fragile materials. Therefore, nowadays, continuum models of damage mechanics are used, similar to smeared crack models, and discrete models such as discrete element methods and molecular dynamics schemes, which present their advantages and disadvantages [4].

In this study, the load capacity of friction and tip piles is analyzed using conventional methods according to the failure mechanisms proposed by Terzaghi and Meyerhof. In addition, two computational models are used. The first is the finite element method using a damage mechanics model and the second is the peridynamic model in which forces between pairs of particles are used.

\section{Analysis of driven piles}

Three types of analysis are presented: the first one is using conventional models such as the ones proposed by Terzaghi and Meyerhof, these models are empirical and semi-empirical, the second one is using a continuum local model, the finite element method, and the third one, a non-local continuum model, the bond-based peridynamic model.

\subsection{Analysis using Bearing Capacity Theories}

By analyzing semi-infinite two-dimensional media using continuum mechanics, solutions were obtained for surface and deep foundations. These solutions were used by Terzaghi and Meyerhof in the development of Soil Mechanics and Geotechnics models, which were adapted for square or circular section foundations. Terzaghi theory uses Prandtl and Reissner solutions formulated in 1921 and 1924 respectively, in which a continuous two-dimensional, semi-infinite, homogeneous and isotropic medium is assumed using plane strain conditions. In this model the theory of plasticity is used in the limit state, assuming a perfectly plastic model. In these theories, Mohr-Coulomb failure envelopes are used in which soil is considered to have cohesion and friction properties between particles.

To solve this problem, the length of the foundation is assumed to be infinite since it is a two-dimensional plane stress problem. Likewise, soil above the foundation depth is replaced with a uniformly distributed load, as shown in [1]. With this model the following formula was obtained for the calculation of the bearing capacity.

$$
q_{u}=C N_{c}+\gamma D_{f} N_{q}+0.6 \gamma R N_{\gamma}
$$

in which, $\mathrm{C}$ is the soil cohesion, $\gamma$ is the soil volumetric weight, $D_{f}$ is the footing depth, $R$ is the foundation radius, and, $N_{c}, N_{q}, N_{\gamma}$ are factors that depend on the soil internal friction angle.

For example, for a tip pile with a foundation depth of $1.5 \mathrm{~m}$, a soil volumetric weight of 19.6 $\mathrm{kN} / \mathrm{m} 3$, a pile diameter of $22.0 \mathrm{~cm}$, a soil internal friction angle the angle of $30^{\circ}$ and the cohesion is null, the bearing capacity can be calculated assuming a local type of failure. For this type of failure the values of $N_{c}, N_{q}, N_{\gamma}$ are 35.0, 22.0 and 20.0 respectively. Using Equation (1) the bearing capacity is $681.1 \mathrm{kN} / \mathrm{m}^{2}$. The total capacity is obtained by multiplying by the transverse area of the pile, so that the bearing capacity is $25.87 \mathrm{kN}$.

If this problem is analyzed as if the pile worked under friction, the equation to obtain the bearing capacity is as follows

$$
q_{f}=0.5 \pi D K_{o} \gamma L^{2} \tan \delta \text {, }
$$

where $\mathrm{D}$ and $\mathrm{L}$ are the diameter and length of the shaft respectively, $\gamma$ is the soil volumetric weight, $K_{o}$ is the soil friction coefficient, which varies between 0.4 and 0.6 , and $\delta=\frac{2}{3} \phi$, in which $\phi$ is the soil internal friction angle. For this example, the value of $\delta$ is $22^{\circ}$, the value of $K_{o}$ is 0.5 and the values of the other variables remain unchanged. Using Equation (2) the bearing capacity 
considering only friction is $3.16 \mathrm{kN}$. By adding both bearing capacities, the total capacity of the pile is $29.03 \mathrm{kN}$ (6.53 kips).

In many cases, loads applied to the piles may have an eccentricity, in this case the pile will work as a beam, and the procedure is described in [5]. The safety factor is obtained using the following expression

$$
F S=\frac{Q}{\sum_{i=1}^{n} R_{i}} \geq 2.5,
$$

in which, $Q$ is the summation of all the horizontal stresses, multiplied by its area along the pile length, and is obtained using

$$
Q=\sum_{i=1}^{n}\left[N_{\varphi} \sigma_{z}+2 C_{i} \sqrt{N_{\varphi}}\right] H_{i} D
$$

where $\sigma_{z}$ is the vertical stress at the midpoint of the stratum $H_{i}, D$ is the pile diameter and $C_{i}$ the cohesion coefficient at stratum $H_{i} . N_{\varphi}$ is a function of the internal friction angle, and is obtained with:

$$
N_{\varphi}=\tan ^{2}\left(45^{\circ}+\frac{\varphi}{2}\right) \text {, }
$$

reactions $R_{i}$ are obtained assuming that the pile is supported vertically as is shown in Figure 1 . Medina et al. [5] shows a procedure to obtain these reactions using the finite element method. In the following sections, these reactions are calculated with the finite element method and the peridynamic model.
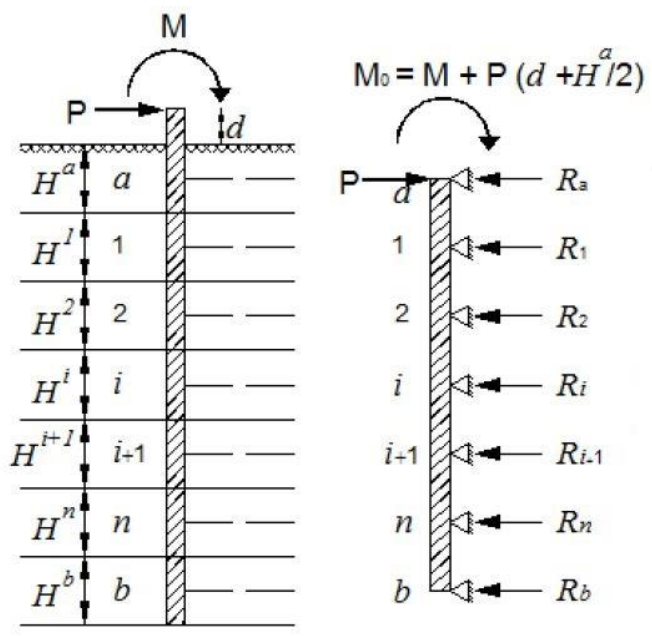

Figure 1. Pile with a vertical load, moment and horizontal reactions [Medina 2013].

\subsection{Analysis using the Finite Element Method}

Discontinuities are present in quasibrittle materials even when they are unloaded. In the model of smeared cracks, the constitutive properties of the material are artificially modified instead of their geometric properties. The previous problem is analyzed using the smeared crack model with the finite element method. To perform the analysis with the finite element, constant strain triangular elements were used (Figure 2). Soil modulus of elasticity considered was $70 \mathrm{MPa}$ with a Poisson ratio of 0.3 , and the modulus of elasticity of the concrete pile concrete was $25 \mathrm{GPa}$ with a Poisson ratio of also 0.3 . The maximum tensile soil stretch is $1.3 \times 10^{-6}$ and in compression is $1.0 \times 10^{-5}$. For concrete tension, the maximum strain is $1.3 \times 10^{-4}$ and $1.0 \times 10^{-3}$ for concrete compression. A total of 78 nodes with two degrees of freedom each and 120 elements in total were used. Two cases were considered, one with a vertical load, and another one with a vertical load and a moment. The moment was simulated using a horizontal load with an eccentricity (Figure 3). Using the finite element method with the smeared crack model, the maximum load obtained is $9.8 \mathrm{kN}$. This load corresponds to the maximum soil shear strength. Since this method is very sensitive to the mesh size, the maximum load corresponds to the creep point of the strain-stress curve. For the case of a vertical load and a moment, safety factors were obtained with equation (3), where horizontal stresses were calculated with equations (4) and (5), assuming a cohesion-less soil $\left(C_{i}=0\right)$. In addition, reactions $\left(R_{i}\right)$ for 
equation (3), were obtained from the horizontal stress $\left(\sigma_{x}\right)$, multiplied by its corresponding width $D$ and height $H_{i}$.

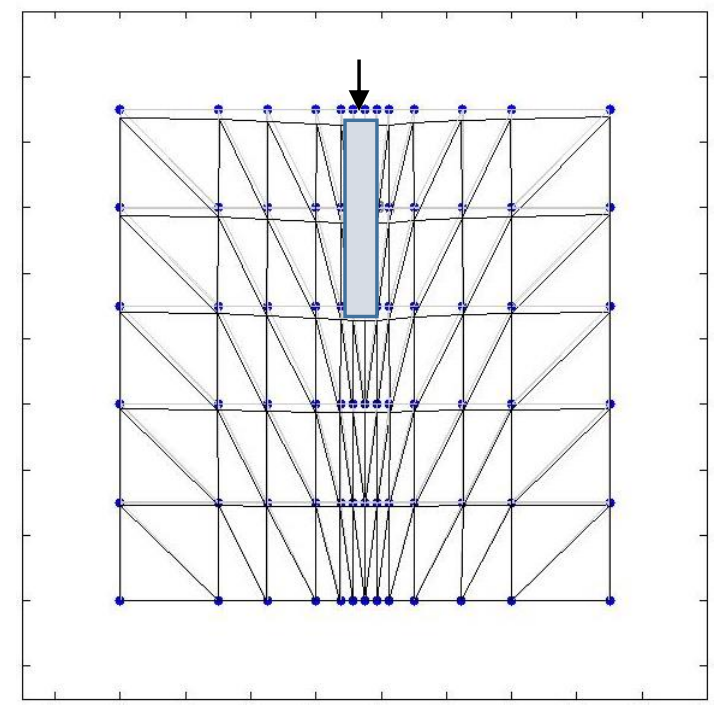

(a)

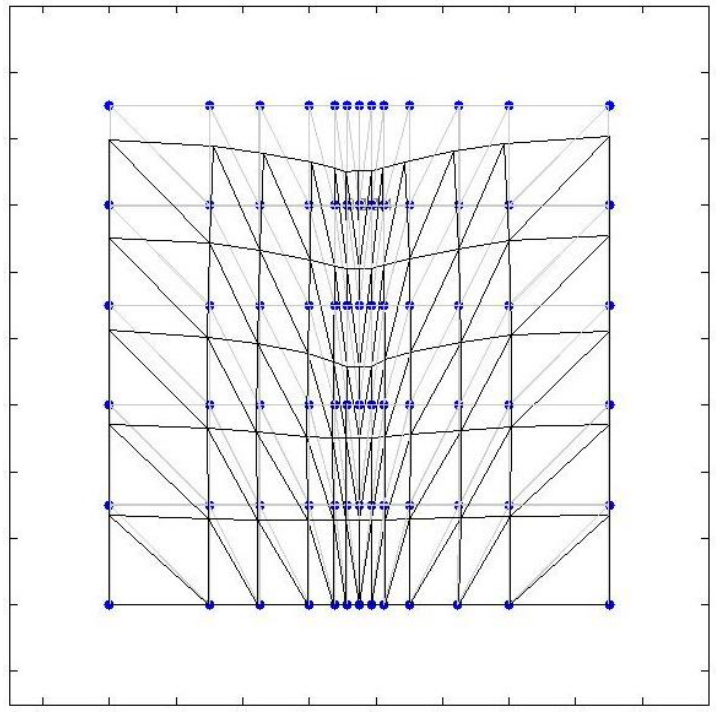

(b)

Figure 2. Pile with a vertical load, using the Finite Element Method: (a) Pile geometry and applied load; (b) Deformed shape at failure.

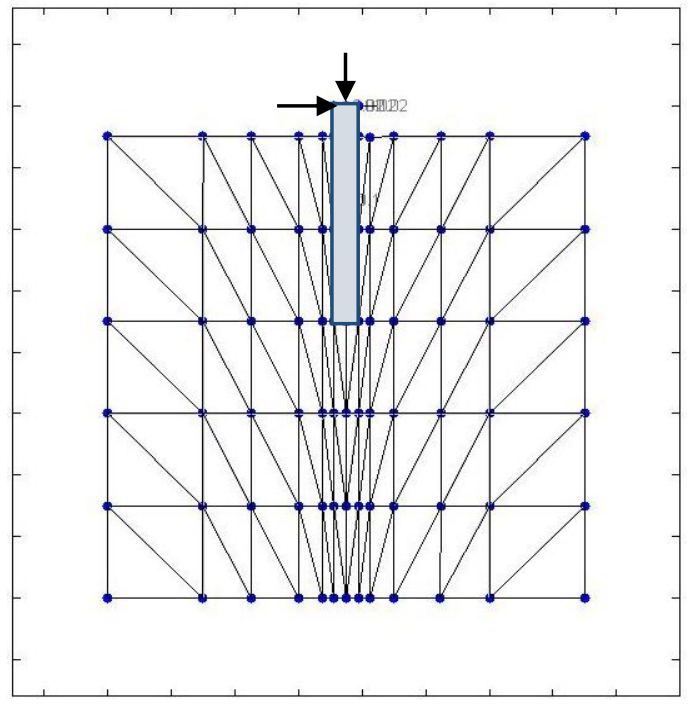

(a)

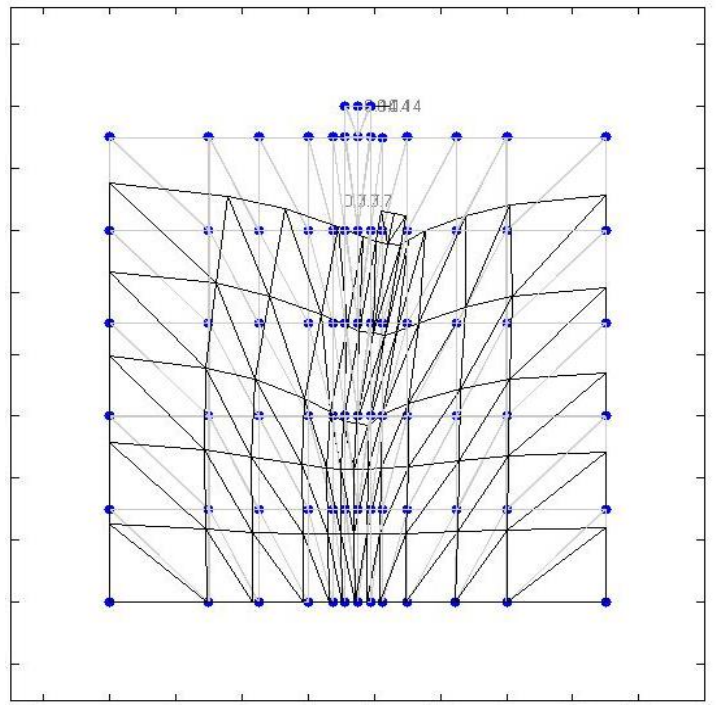

(b)

Figure 3. Pile with a vertical load and a moment, using the Finite Element Method: (a) Pile geometry and applied load; (b) Deformed shape at failure.

\subsection{Analysis using the Peridynamic Model}

In the peridynamic formulation a vector function that represents the interaction between pairs of particles is postulated. This function depends on the force and its units are force by unit volume squared. The force is supposed to be a function of the relative position and the relative displacement between the particles of an elastic material. It is also assumed that there is a positive value called the material horizon in which the particles outside this horizon do not interact. For soil and concrete 
particles the constitutive material model is shown in Figure 4, in which material behaves linearly before a maximum stretch $\left(S_{\text {tens }}\right)$, and a negative stretch called $\left(S_{\text {comp }}\right)$, these values are functions of the relative displacements between particles. Also a plateau is specified $\left(\alpha_{\text {tens }} S_{\text {tens }}, \alpha_{\text {comp }} S_{\text {comp }}\right)$ in which particles can stretch with no change in the pair-wise force function. Force between particles drops to zero for stretches larger than $\alpha_{t e n s} S_{\text {tens }}$ and less than $\alpha_{\text {comp }} S_{\text {comp }}$.

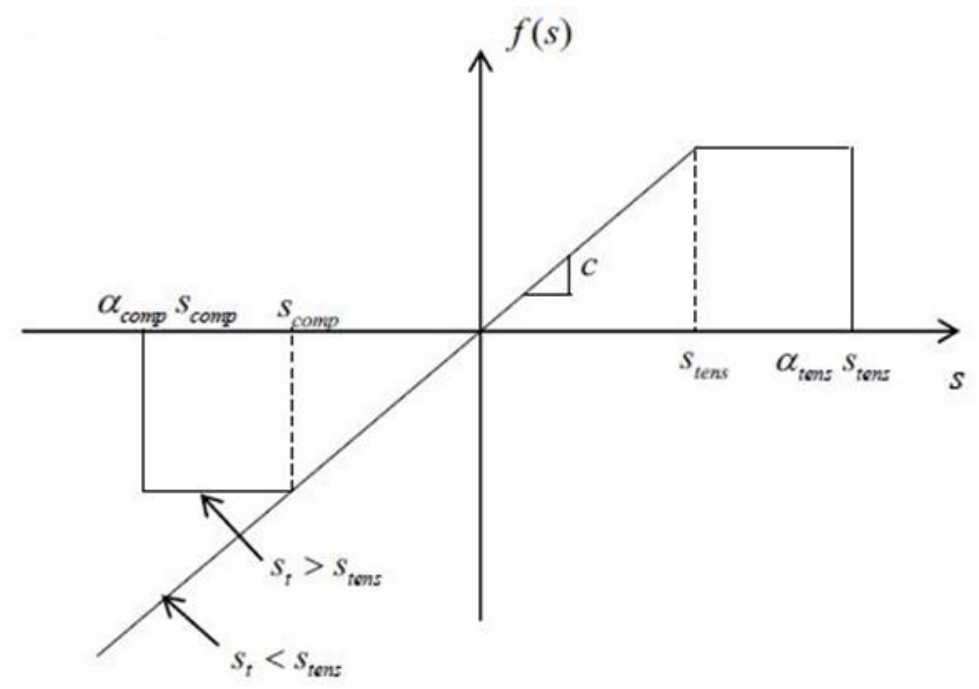

Figure 4. Constitutive material model for the Peridynamic formulation.

Using the peridynamic model, a computational model was developed in which particles are distributed in an array, where dynamic equilibrium equations are satisfied. With this model the problem of a tip pile subject to a vertical load is analyzed in which load is increased monotonically. Likewise, the same material properties used in the finite element example were chosen. In Figure 5 the pile model subjected to a vertical pressure is shown using the peridynamic model, where broken links in tension are shown in black, crushed links in compression in black, damaged links in the plateau $\boldsymbol{\alpha}_{\text {tens }} \boldsymbol{S}_{\text {tens }}$ are in gray and in compression $\left(\boldsymbol{\alpha}_{\text {comp }} \boldsymbol{S}_{\text {comp }}\right)$ are shown also in gray. With this model the maximum load obtained for a creep state is approximately $9.8 \mathrm{kN}$.
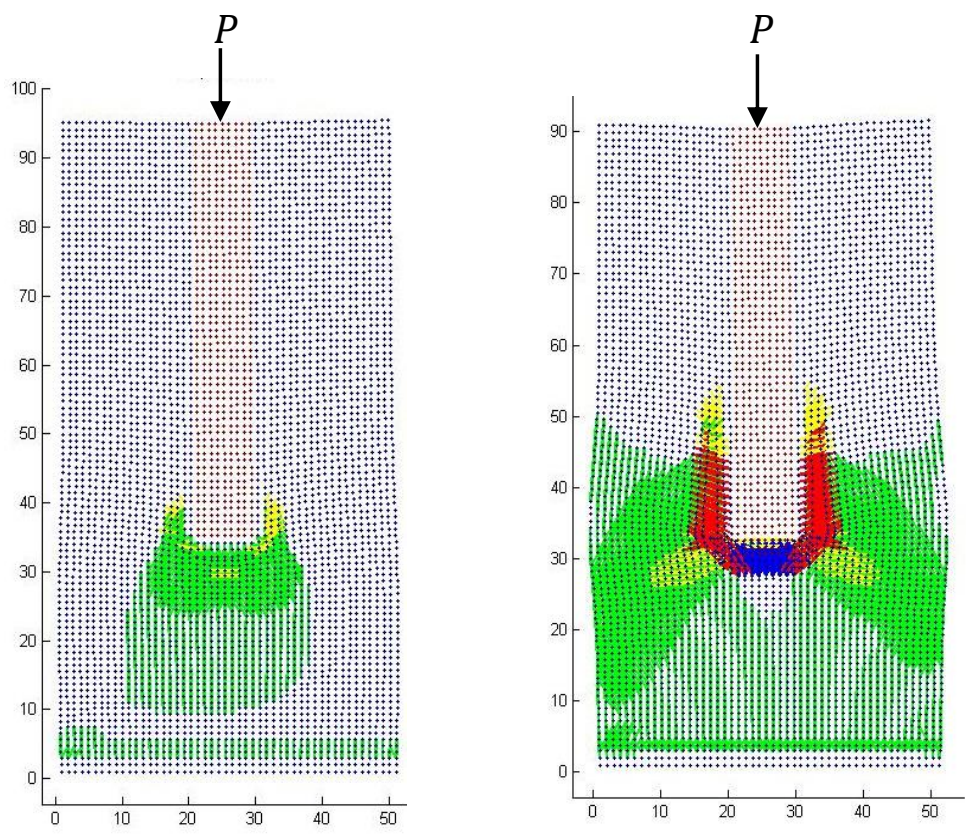

Figure 5. Pile with a vertical load, using the Peridynamic Model at different loads. 
Similar to the finite element method, a pile was analyzed with a vertical load and a moment as shown in Figure 6. Safety factors can also be obtained, horizontal stresses in the numerator of equation 3 are calculated in the same form as the in finite element method, for the denominator, reactions are calculated also using $\sigma_{x}, D$ and $H_{i}$. Nevertheless, the stress tensor at each is obtained with a summation of forces inside the material horizon at different directions, this methodology is described in [6]. Figure 7 shows the safety factor for the finite element method and the peridynamic model for different applied loads, in which the moment is proportional to the vertical load.
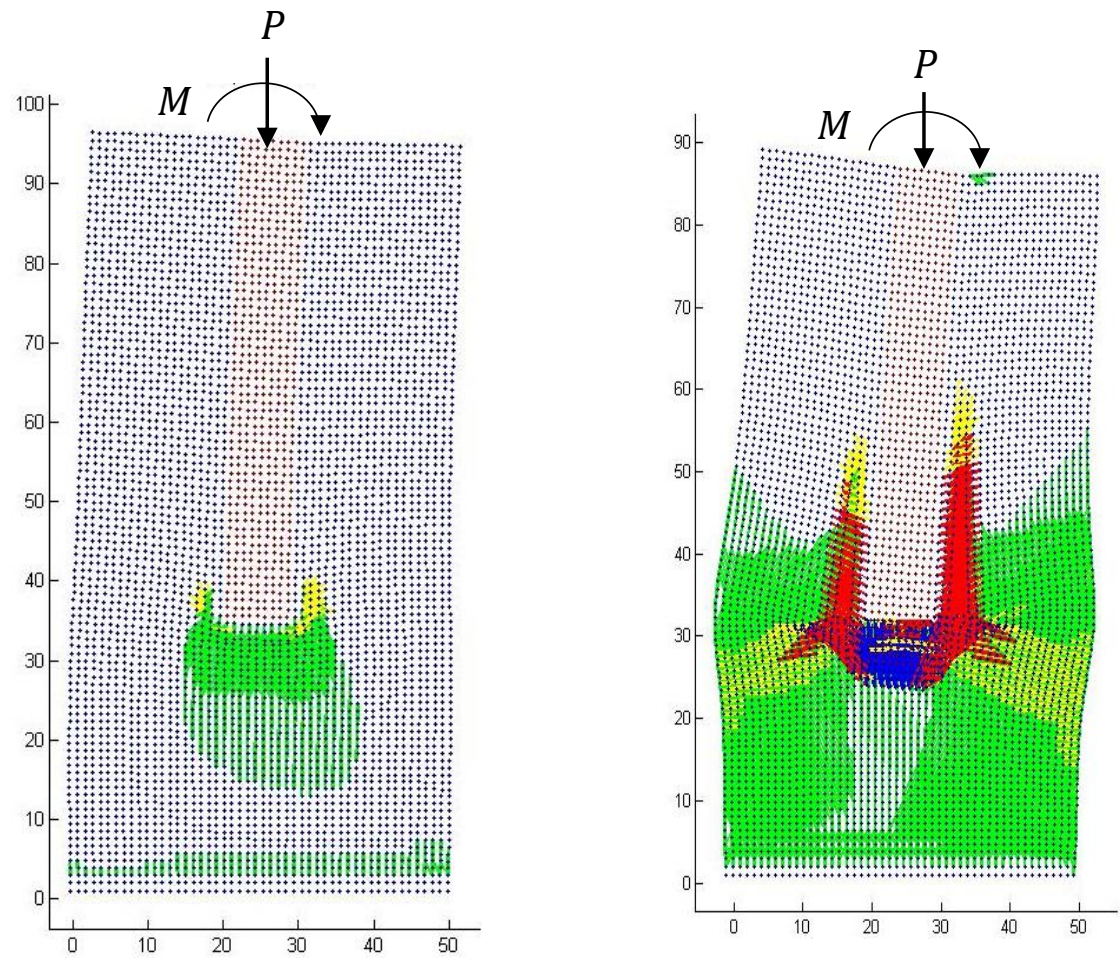

Figure 6. Pile with a vertical load and a moment, using the Peridynamic Model at different loads.

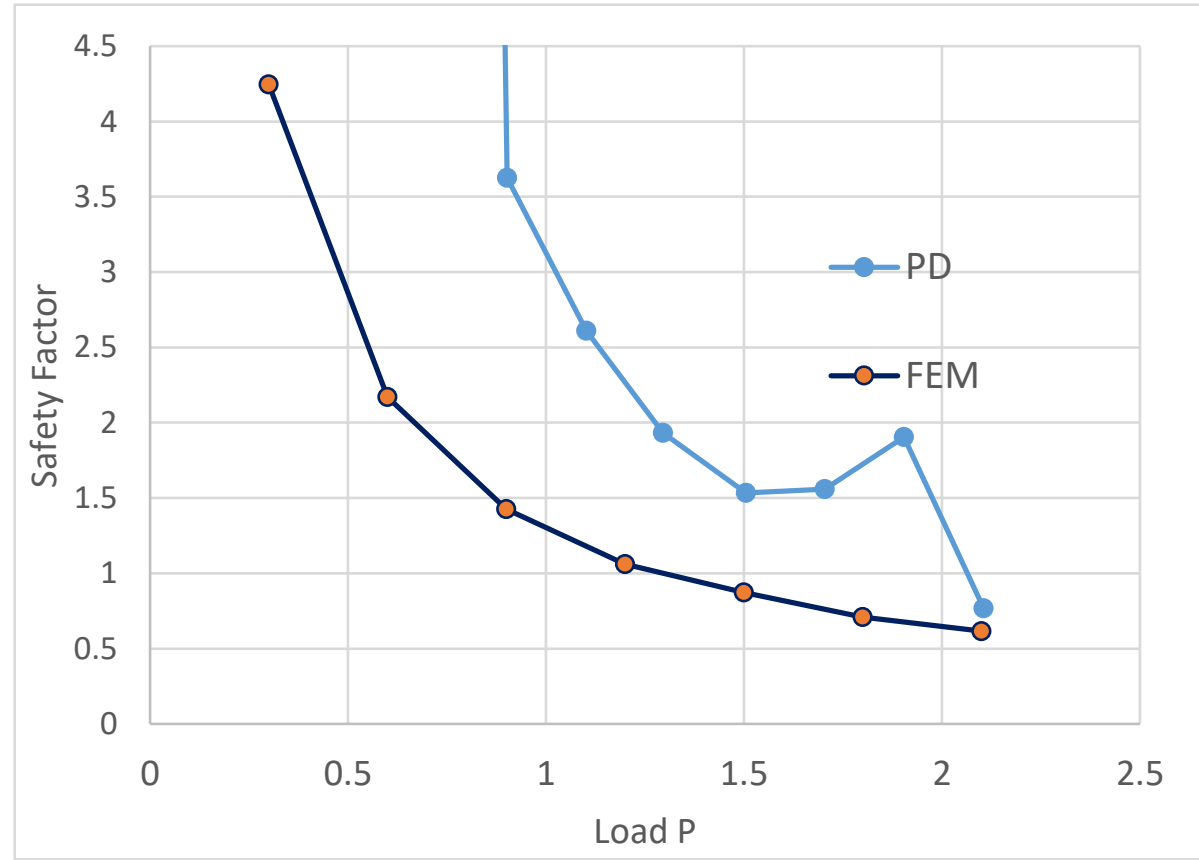

Figure 7. Safety factors using the Finite Element Method and the Peridynamic Model. 


\section{Conclusions}

In this work, the study of the bearing capacity of foundation piles subjected to vertical loads was performed. Current design methods are based on Terzaghi and Meyerhof theories. These models were formulated at the beginning of the past century and are based on theories of elasticity and perfect plasticity models. Nevertheless, there are strong hypotheses in the development of these theories such as continuity and perfect failure surfaces.

With the advent of the computer age there has been an advent of mechanistic methods for many civil engineering problems, both in soil mechanics and in structural mechanics. Most popular methods are the finite element, discrete element methods and smeared crack models.

By performing the analysis with the finite element method and the smeared crack model in its linear portion, it was found that the pile resistance was around $9.8 \mathrm{kN}$. This value is far from the values obtained with the semi-empirical models of Terzaghi and Meyerhof. One possible reason for this difference is that the maximum load in the linear finite element method is quite far from the ultimate load in the theories of Terzaghi and Meyerhof, in which plastic failure envelopes are used.

In an effort to unify continuum non-linear models and particle models, the peridynamic model was proposed. The peridynamic model is a continuous version of molecular dynamics models where discontinuities are part of the formulation. However, using particles has the disadvantage of using a large amount of computational resources.

Using the peridynamic method to the analysis of piles, a maximum load of approximately 9.8 $\mathrm{kN}$ was obtained at the creep level, very similar to the value obtained with the smeared crack model. However, it was observed that the pile remained stable for higher loads, where areas of plasticity and discontinuities in the soil mass were obtained. However, no failure envelopes could be observed as predicted in the Terzaghi and Meyerhof models.

Therefore, it is important that future research on mechanistic models unify continuum theories and particle models. It is also necessary to conduct mesh convergence studies to be able to make a complete verification of these models. In addition to this, three-dimensional mechanistic models and physical experimentation should be incorporated into these studies.

\section{References}

1. Juarez, B.; Rodríguez, R., Soil Mechanics, volume II, 2nd ed.; Limusa Editors, Mexico, 1996; pp. 459-477.

2. Sau, N., Peridynamic Model of Quasibrittle Structures. PhD Thesis, University of New Mexico, Albuquerque, New Mexico, 2008.

3. Silling, S. A., Reformulation of elasticity theory for discontinuities and long-range forces. J. Mech. Phys. Solids 2000, 48, 175-209.

4. Bazant, Z. P.; Lin, F. B., Non-local smeared cracking model for concrete. J. Eng. Mech. 1988, 114(10), 2493-2510.

5. Medina, J.; Sau, N.; Acuña, Q.; Quintana, J., Stability Analysis of Piles Subjected to Lateral Load in Static and Seismic Conditions. J.G.R.E. 2013, 1, 11-24.

6. Sau, N; Medina, J; Borbon, A.C. Peridynamic Modelling of Reinforced Concrete Structures. Eng. Fail. Anal. 2019, 103, 266-274. 